\title{
LES PERTURBATIONS INDUITES PAR PARASITORHABDITIS IPSOPHILA (NEMATODA : RHABDITIDAE) dans le tube digestif d'Ips sexdentatus (Insecta : Scolytidae)
}

\author{
F. LIEUTIER* (1) \\ Collaboration technique : M. Jastrabsky et P. Bonnafe
}

RÉSUMÉ. L'étude histologique du tube digestif des Ips sexdentatus parasités par Parasitorhabditis ipsophila montre une destruction importante des microvillosités et une vacuolisation des cellules de l'épithélium intestinal, dans la partie terminale du mésentéron. Aucun dégât n'est observé dans l'intestin postérieur ou lors de faibles contaminations. Parallèlement, l'étude biochimique indique une diminution de la quantité de protéines dans le bol alimentaire des insectes parasités, en même temps qu'une augmentation dans la paroi intestinale. Les dégâts semblent essentiellement mécaniques et expliquent les perturbations observées précédemment au niveau des populations. La contradiction apparente entre l'importance des dégàts au niveau de l'épithélium intestinal et les faibles conséquences du parasitisme pour les populations hôtes peut s'expliquer par la localisation préférentielle des nématodes dans la partie terminale du mésentéron et la partie proximale de l'intestin postérieur.

The perturbations caused by Parasitorhabditis ipsophila (Nematoda : Rhabditidae) in the digestive tract of Ips sexdentatus (Insecta : Scolytidae)

SUMMARY. The histological study of the digestive tract in Ips sexdentatus parasitized by $P$. ipsophila showed major destruction of the microvillosities as well as vacuolization of the epithelial cells in the terminal part of the mesenteron. No damage could be observed in the hind gut nor during low contamination. Concurrently, the biochemical study indicated a lower amount of proteins in the alimentary bolus of parasitized insects along with a higher amount of proteins in the intestinal wall. Damage seemed chiefly mechanical and accounted for the disorders previously observed in populations. The apparent contradiction between the magnitude of damage to the intestinal epithelium and the mild effects of parasitism on host populations might be explained by the preferential location of nematodes in the terminal part of the mesenteron and the proximal part of the hind gut.

- Institut national Agronomique, Laboratoire de Zoologie, 16 rue Claude-Bernard, F 75005 Paris.

(1) Adresse actuelle : INRA, Station de Zoologie et de Biocénotique forestière, Centre de Recherche Forestière d'Orléans, Ardon, F 45160 Olivet.

Accepté le 2 février I984. 
Parasitorhabditis ipsophila, parasite très fréquent du tube digestif des adultes d'I ps sexdentatus, paraît n'avoir qu'une action limitée sur les populations de son hôte (Lieutier, 1984) : léger retard dans l'essaimage et le démarrage de la ponte ; faible diminution de la densité des encoches de ponte et des œufs. Néanmoins, il importe de déterminer les modalités de cette action ; nous nous proposons donc d'examiner les perturbations causées par le parasite au niveau du tube digestif de l'insecte, d'un point de vue histologique et biochimique.

Les perturbations provoquées par les nématodes du tube digestif chez les insectes ont été peu étudiées. Les cas les plus fréquents de contamination concernent les nématodes à cycles hétéroxènes (Spiruroïdea, Seuratoïdea) dont les insectes représentent l'hôte intermédiaire, l'hôte définitif étant un Vertébré ainsi que les Oxyures dont l'hôte unique et obligatoire est un insecte ou un vertébré.

Comme le signale Welch (1965), les Oxyures ne sont en général pas pathogènes pour les insectes et si la mort survient parfois, elle est due probablement à des lésions du tube digestif, qui provoquent la pénétration de microorganismes pathogènes dans la cavité générale. Ainsi, Taylor (1968) observe dans le tube digestif de Leucophaea maderae, des lésions provoquées sans doute par l'activité du nématode Leidynema, sans aucune conséquence pour la vie de l'insecte. Poinar (1973) signale que la croissance et la multiplication de Thelastoma pterygoton provoquent également des lésions dans le tube digestif d'Oryctes sp. Par contre, Rostrom (1966) note simplement une fragmentation de la membrane peritrophique chez Hydrous aculeatus parasité par Galebiella sp.

Contrairement aux Oxyures qui ne pénètrent normalement pas dans la paroi du tube digestif de l'insecte, les Spirurides et les Seuratides effectuent toujours une migration plus ou moins importante à l'intérieur de l'épithélium digestif de leur hôte intermédiaire. On note, dans ce cas, une destruction locale des cellules de l'épithélium et leur désorganisation puis la formation, autour du parasite, de capsules tissulaires dues à l'hypertrophie des cellules épithéliales se constituant en syncytium. C'est le cas en particulier pour les Physalopteridae (Schell, 1950, Seureau, 1973, Poinar et Hess, 1974), les Rictulariidae (Oswald, 1958, Seureau, 1972, 1973) et les Seuratidae (Quentin et Seureau, 1975) tous parasites de Blattes ou de Criquets. Pour les Seuratidae cependant la capsule est de nature hémocytaire.

Chez les Rhabditoidea, les observations sont encore plus rares. Pour les Diplogasteridae, Merril et Ford (1916) signalent que la pullulation de Diplogasteritus labiatus dans le tube digestif du Cérambycide Saperda tridentata empêche celui-ci d'assurer ses fonctions naturelles et peut provoquer la rupture de la paroi intestinale, puis la mort de l'hôte. Weiser (1966 in Benham, 1974) indique aussi que la présence de Diplogasteridae dans le tube digestif des larves de certains Lépidoptères provoque leur mort. Poinar (1969) a observé que l'infestation expérimentale du tube digestif de Galleria melonella par Mesodiplogaster lheritieri et Pristionchus sp. entraîne la mort de l'hôte, par rupture de la paroi intestinale et invasion de l'hémocoele par les Nématodes et les microorganismes.

Pour les Rhabditidae Ruhm et Chararas (1957) signalent que Parasitorhabditis hectographi provoque chez le Scolytide Dryocoetes hectographus, l'amincissement de 
l'épithélium intestinal, sa vacuolisation et la disparition des microvillosités. Nickle (1963) note également une réduction de l'épithélium du tube digestif des Ips confusus parasités par un Parasitorhabditis. Fuchs (1915) chez I. typographus et Blinova et coll. (1980) chez I. subelongatus indiquent que, dans certains cas d'infestations très lourdes, l'obstruction du tube digestif par les nématodes peut aboutir à la mort de l'hôte. Wahab (1962, in Passera, 1975) observe que l'infestation des glandes pharyngiennes des Fourmis par Diploscapter lycostoma, Pristionchus lheritieri ou Eudiplogaster histophorus provoque l'amincissement de l'épithélium, une moins grande densité du cytoplasme et la perte du rôle sécréteur de ces glandes. Markin et Mc Coy (1968, in Poinar, 1972 et Passera, 1975) font des observations analogues chez Iridomyrmex humilis parasité par Diploscapter lycostoma.

\section{I - Méthodes et techniques}

\section{I.1. - Obtention des insectes}

Les insectes parasités sont récoltés directement sur le terrain en Forêt de Fontainebleau ou d'Orléans. En revanche, par suite du taux de parasitisme très élevé dans la nature, il est nécessaire de procéder par élevage pour obtenir les insectes non parasités : la contamination pouvant avoir lieu au stade larvaire, l'élevage est mis en route à partir d'œufs récoltés sur le terrain et installés sur papier filtre humide. Dès l'éclosion, les larves sont placées individuellement dans une petite cavité creusée dans le liber d'un morceau d'écorce fraîchement détaché d'un rondin sain. Le fragment d'écorce est alors placé entre deux plaques de verre, deux bandes de papier filtre permettant une humidification toutes les 24 à 48 heures. L'écorce est changée toutes les semaines jusqu'à ce que le stade adulte à coloration brune soit atteint. Les insectes sont alors placés sur des rondins sains dans lesquels ils pénètrent. Ils en ressortent au moment de l'essaimage et sont installés pour pondre directement sur d'autres rondins. Des générations successives d'insectes non parasités peuvent alors être obtenues sans passer par l'intermédiaire de l'élevage entre plaques de verre.

\section{I.2. - Techniques histologiques}

Les insectes sont disséqués dans la solution de Ringer ou directement dans le fixateur, le tube digestif est fixé dans le liquide de Carnoy pendant 2 heures ou le bouin alcoolique pendant 12 heures. Après déshydratation par l'alcool éthylique et inclusion à la paraffine, les organes sont coupés à 5 ou $7 \mu \mathrm{m}$ d'épaisseur. Les colorations utilisées sont le glychémalum-érythrosine, l'azan de Heidenhaim ou l'hémaluméosine-vert lumière.

\section{I.3. - Techniques biochimiques}

Environ 50 à 100 insectes, selon les catégories, sont disséqués dans la solution de Ringer. Les tubes digestif (intestin moyen) sont posés sur une lame de verre et 
leur contenu est séparé de la paroi. Les deux parties sont immédiatement placées à $4^{\circ} \mathrm{C}$ où elles sont directement regroupées par catégorie, puis broyées dans un microbroyeur de Thomas avec un volume connu d'eau distillée. Le broyat est ensuite laissé 1 heure entre $0^{\circ}$ et $4^{\circ} \mathrm{C}$ pour permettre la diffusion des substances. Les dosages sont effectués, à raison de 3 à 6 répétitions par catégorie, sur un volume de paroi de tube digestif correspondant à 1 ou 2 insectes et sur un volume de contenu de tube digestif correspondant à 7 insectes. Seule la quantité totale de protéines est mesurée, grâce au réactif de Folin-Ciocalteus, selon la méthode de Lowry et coll. (1951) modifiée par Parker (1971). Les densités optiques sont lues à 750 nanomètres, au spectrophotomètre Beckman Model 24, la courbe standard ayant été établie avec la Serum albumine bovine.

\section{II — Résultats}

\section{II.1. - Étude histologique}

La partie terminale du mésentéron et la partie proximale de l'intestin postérieur, où se localisent préférentiellement les nématodes (Lieutier, 1984), ont été plus particulièrement étudiées.

II.1.1. - Le mésentéron. Il se caractérise chez les insectes sains par l'existence de cellules bien accolées les unes aux autres et fixées sur une membrane basale importante. Ces cellules, sans vacuolisation apparente, possèdent des microvillosités bien développées formant une bordure en brosse très régulière ( $f i g .1)$. Par endroits s'intercalent des zones de régénération et des zones de sécrétion.

Chez les insectes fortement parasités par P. ipsophila la bordure en brosse est très irrégulière et les microvillosités sont réduites, parfois même non visibles et on note une vacuolisation plus ou moins importante des cellules ( $f i g$. 2). Les nématodes sont localisés le plus souvent dans la lumière du tube digestif mais il n'est pas rare de les observer aussi entre les cellules épithéliales, atteignant parfois la membrane basale (fig. 3). Cette action provoque la dissociation des cellules et probablement la destruction de certaines d'entre elles. La membrane basale ne semble par contre pas abîmée et jamais nous n'avons observé de passage de nématodes dans la cavité générale. En cas de faible infestation, aucun dégât ne peut être observé.

Dans la partie antérieure du mésentéron, on n'observe pas de dégâts, quel que soit le niveau de l'infestation.

II.1.2. - L'intestin postérieur. Dans cette zone, aucune modification de la paroi intestinale n'a pu être mise en évidence, même en cas d'infestation très forte (fig. 4).

\section{II.2. - Étude biochimique (tableau I)}

Les nématodes semblent provoquer une augmentation de la quantité totale de protéines dans l'épithélium intestinal et une diminution dans le bol alimentaire, mais les différences ne sont pas très importantes. 

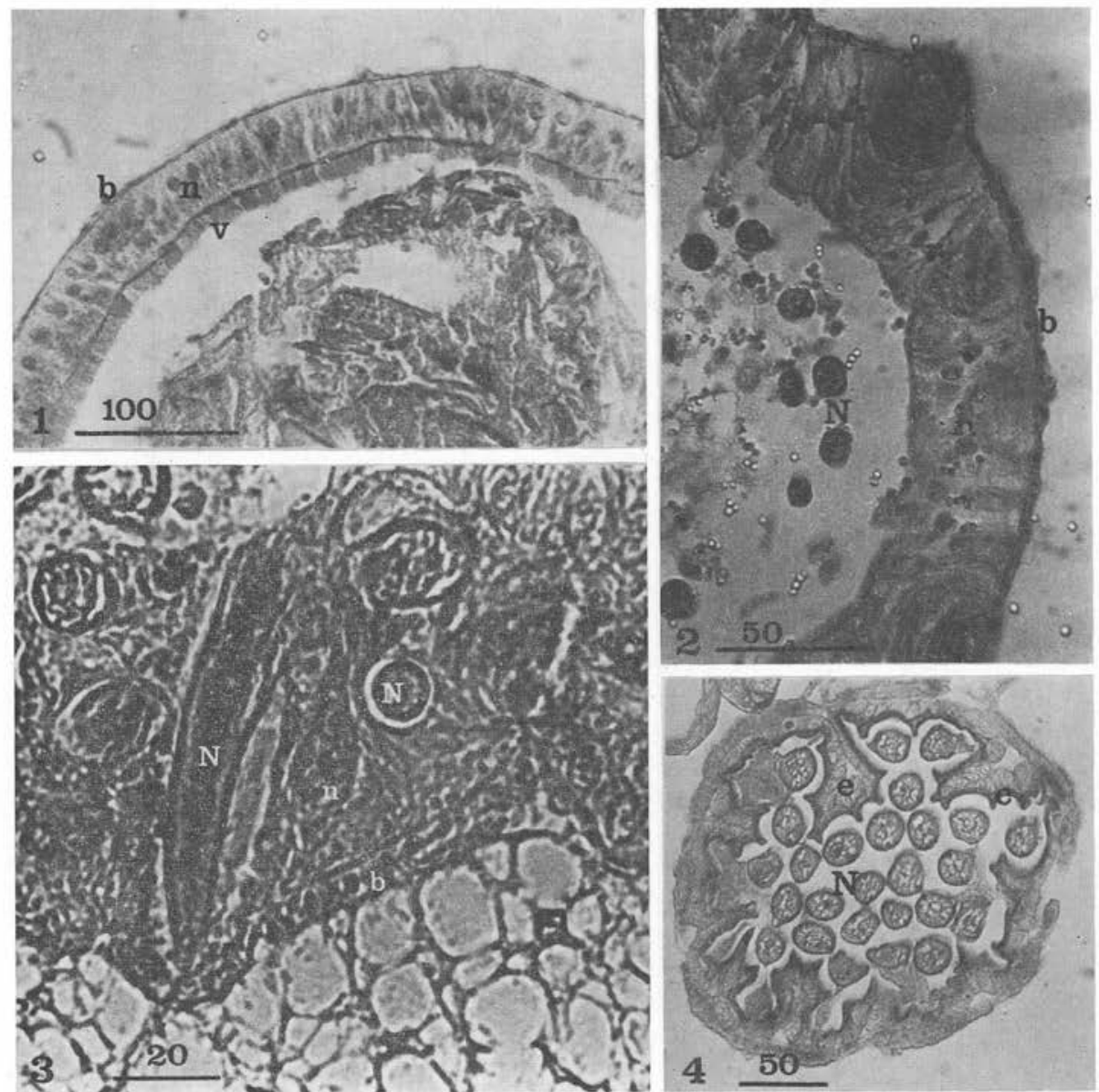

FIG. I. - Épithélium de la partie postérieure du mésentéron d'un adulte d'Ips sexdentatus non parasité. $\mathrm{b}=$ membrane basale $; \mathrm{n}=$ noyau ; $\mathrm{v}=$ microvillosités. L'unité de longueur est le micron. Fixation: Bouin ; coloration : glychémalum erythrosine.

FIG. 2. - Épithélium de la partie postérieure du mésentéron d'un adulte d'Ips sexdentatus fortement parasité par Parasitorhabditis ipsophila. Les flèches indiquent la forte réduction des microvillosités. $\mathrm{b}=$ membrane basale $; \mathrm{n}=$ noyau; $\mathrm{N}=$ nématodes. L'unité de longueur est le micron. Fixation $=$ Bouin $;$ coloration $=$ glychémalum-érythrosine.

FIG. 3. - Pénétration des larves de Parasitorhabditis ipsophila dans l'épithélium de la partie postérieure du mésentéron d'Ips sexdentatus fortement parasité. b = membrane basale; $\mathrm{n}=$ noyau ; $\mathrm{N}=$ nématodes. L'unité de Jongueur est le micron. Fixation : Carnoy ; coloration : glychémalum-érythrosine.

FIG. 4. - Intestin postérieur d'un adulte d'Ips sexdentatus fortement parasité par Parasitorhabditis ipsophila. $\mathrm{c}=$ cuticule $; \mathrm{e}=$ épithélium ; $\mathrm{N}=$ nématodes. L'unité de longueur est le micron. Fixation : Bouin ; coloration : Azan. 


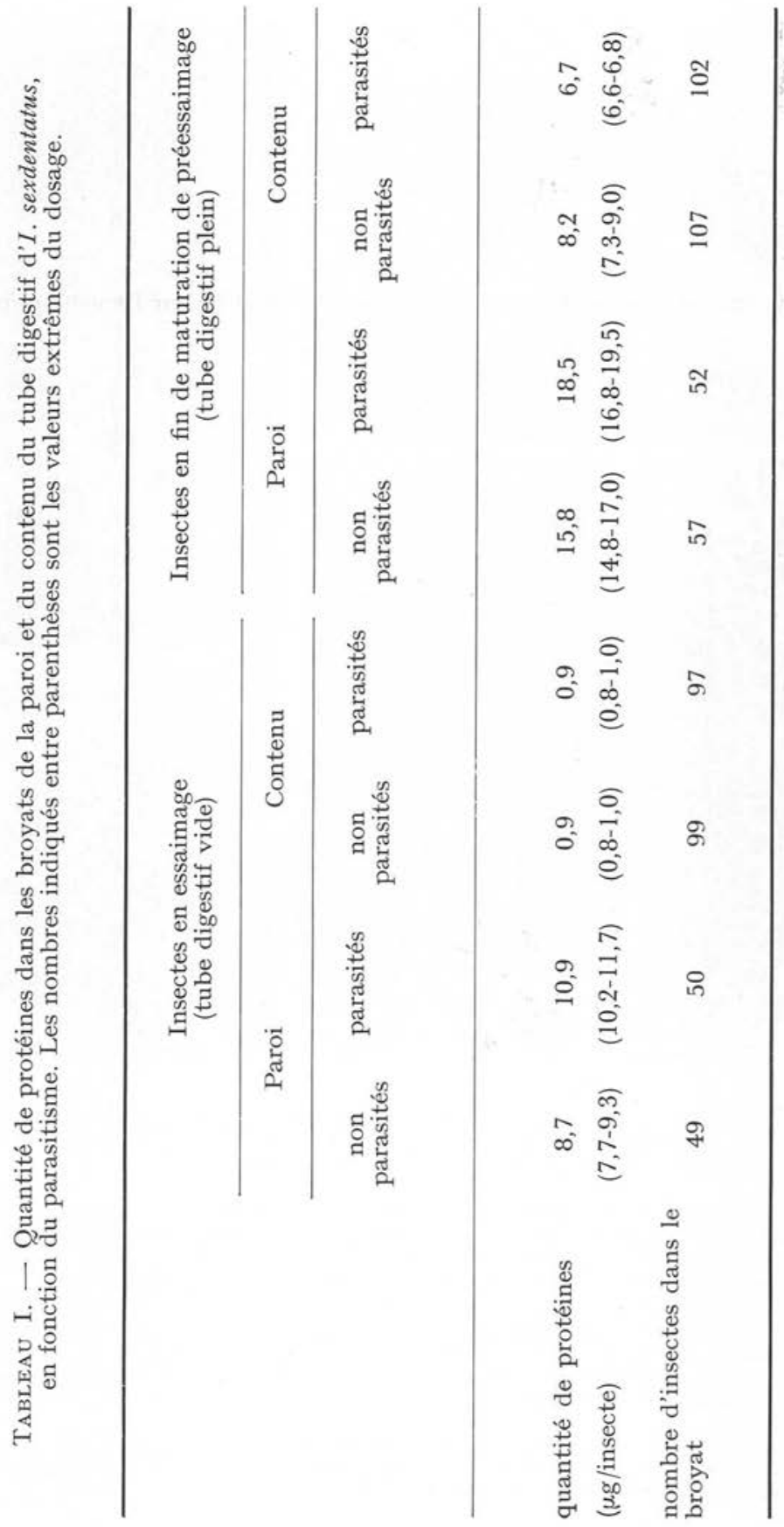




\section{III - Interprétation et discussion}

P. ipsophila cause des dégâts mécaniques importants dans l'épithélium intestinal de son hôte, en particulier au niveau des microvillosités. Ces observations sont comparables à celles de Ruhm et Chararas (1957) chez D. hectographus parasité par $P$. hectographi, qui ont noté aussi une destruction de la bordure en brosse et une vacuolisation des cellules épithéliales, et même un amincissement de l'épithélium. Nickle (1963) rapporte aussi l'action d'un Parasitorhabditis sur l'épithélium intestinal d'Ips confusus. Étant donné le rôle joué par les microvillosités dans l'absorption intestinale, la présence des larves de $P$. ipsophila en quantité importante a sans doute des conséquences sur l'assimilation des nutriments par l'insecte. La destruction mécanique des microvillosités est donc probablement une explication aux perturbations observées dans le développement des Scolytes très infestés (Lieutier, 1984).

Cette destruction n'est cependant peut-être pas le seul mode d'action des parasites. Ainsi, les résultats des dosages de protéines suggèrent une action spoliatice par prélèvement sur le bol alimentaire de l'hôte. $P$. ipsophila pourrait donc s'alimenter dans l'insecte, bien qu'il ne semble pas y évoluer. Le prélèvement reste cependant très faible et n'a sans doute que peu de conséquences pour le Scolyte. Par contre, il est difficile d'expliquer que le parasitisme cause une légère augmentation de la quantité de protéines dans la paroi du mésentéron ; peut-être s'agit-il d’un signe de dégénérescence des cellules ou d'un blocage des enzymes digestives qui ne seraient plus secrétées. Par ailleurs, il est possible que la vacuolisation des cellules épithéliales soit due à l'intervention de substances chimiques sécrétées par le nématode, mais on peut aussi l'interpréter comme la conséquence de la destruction des microvillosités et le signe de la mort prochaine des cellules. En définitive, si elle n'est sans doute pas la seule à intervenir, la destruction mécanique de l'épithélium, qui accompagne les mouvements et les déplacements des nématodes, apparaît comme la cause initiale et principale des perturbations déjà signalées.

Cependant, il peut sembler paradoxal que les dégâts rapportés ici au niveau intestinal ne provoquent que des perturbations minimes au niveau des populations de Scolyte (Lieutier, 1984), et donc sans doute aussi minimes au niveau de la physiologie de l'individu-hôte. L'explication réside selon nous dans la localisation préférentielle des parasites. Les larves de $P$. ipsophila se concentrent en effet dans la partie terminale du mésentéron et la partie proximale de l'intestin postérieur. Or, dans cette dernière partie, aucune action mécanique n'est possible (cf. supra), sans doute à cause de la cuticule protectrice de l'intestin postérieur. Les dégâts se produisent donc dans la partie terminale de l'intestin moyen, d'autant plus que leur origine essentiellement mécanique leur confère obligatoirement une localisation précise et les limite à la zone dans laquelle se trouvent les nématodes. Dans les parties antérieures du mésentéron les dégâts sont absents par suite du très faible nombre de nématodes qui s'y trouvent. Les trois quarts de la zone d'absorption restent donc fonctionnels, ce qui doit se traduire par des répercussions physiologiques minimes, même si les dégâts sont localement importants. Il en découle alors naturellement des conséquences 
réduites au niveau du comportement des Scolytes et au niveau de leurs populations (Lieutier, 1984). C'est sans doute la raison pour laquelle un taux très important de contaminations a pu se produire et se maintenir, de telle sorte que l'état non parasitaire constitue l'exception et l'état parasitaire la règle.

Remerciements. L'auteur remercie Monsieur C. Chararas, Directeur de Recherches au CNRS, membre de l'Institut, d'avoir bien voulu relire et critiquer le manuscrit. Il remercie également Messieurs Bonnaire et Tendron, chefs de Centre, Corbaz, Grillo, Lerouet, Trochesec, Vayssette et Vincq, chefs de secteurs, de l'Office National des Fo1êts, ainsi que Monsieur Rousseau, technicien au Service Régional d'Aménagement Forestier de la région centıe, pour l'aide qu'ils lui ont apporté lors de ses études sur le terrain et pour la fourniture du matériel nécessaire aux élevages.

\section{BIBLIOGRAPHIE}

Benham G. S., JR : A synopsis of the obligate and facultative insect parasitic nematodes. $J$. Invert. Pathol., 1974, 24, 263-270.

Blinova S. L., Korenchenko E. A., Ivanova E. C. : (En russe.) Essai d'élevage de scolytes dépourvus d'Helminthes et contamination expérimentale d'Ips subelongatus par différentes espèces de nématodes. Les Helminthes des insectes, Moscou, 1980, 18-21.

Fuchs A. G. : Die Naturgeschichte der Nematoden und einiger anderer parasiten 1 . des $I p s$ typographus - 2. des Hylobius abietis. Zool. Jahrb. (syst), 191 5, 38, 109-222.

LIeUTIER F. : Observations sur le parasitisme d'I ps sexdentatus (Insecta : Scolytidae) par Parasitorhabditis ipsophila (Nematoda : Rhabditidae). Ann. Parasitol. Hum. Comp., 1984, 99 ,

S07-520.

Lowry D. H., Rosebrough N. J., Farr A., Randall R. J. : Protein measurement with the Folin phenol reagent. J. Biol. Chem., 1951, 193, 265-275.

MERRIL J. H., Ford A. L. : Life history and habits of two new nematodes parasitic on insects. J. Agric. Res., I9I6, 6, II 5-127.

NickLe W. R. : Observation on the effects of Nematodes on Ips confusus (Leconte) and other bark beetles. J. Insect. Pathol., 1963, s, 386-389.

Oswald V. H. : Studies on Rictularia coloradensis Hall, I916 (Nematoda : Thelaziidae). I. larval development in the intermediate host. Trans. Am. Microsc. Soc., 1958, 77, 229-240.

PARKER R. : A comparison of haemolymph proteins in two species of Leptinotarsa beetles. J. Insect Physiol., 1971, 17, 1689-1698.

PASSERA L. : Les fourmis hôtes provisoires ou intermédiaires des helminthes. Année Biol., I975, $14,227-259$.

PoINAR G. O. : Diplogasterid nematodes (Diplogasteridae : Rhabditidae) and their relationship to insect desease. J. Invert. Pathol., 1969, I3, 447-454.

Poinar G. O. : Nematodes as facultative parasites of insects. A Rev. Entomol., I972, I7, I03-122.

PoINAR G. O. : Description and observations on a cuticular infection of Thelastoma pterygoton sp. $\mathrm{n}$. (Thelastomatidae, nematoda) from Oryctes spp. (Scarabaeidae: Coleoptera). Proc. Helm. Soc. Wash., I973, 40, 37-42.

Poinar G. O., Hess R. : An ultrastructural study of the response of Blatella germanica (Orthoptera : Blattidae) to the nematode Abbreviata cancasica (Spirurida: Physalopteridae). Int. J. Parasitol., 1974, 4, 133-138.

Quentin J. C., Seureau C. : Sur l'organogenèse de Seuratum cadarachense Desportes, I947 (Nematoda: Seuratoïdea) et les réactions cellulaires de l'insecte Locusta migratoria, hôte intermédiaire. Z. Parasitenk., I975, 47, 55-68. 
Rostrom, Z. M. F. : On a parasitic nematode, Galebiella sp. Basir, 1941, from aquatic beetles in Egypt. J. Helminthol., 1966, 40, I41-146.

RÜHM W., CHARARAS C. : I. Einige neue mit Dryocoetes hectographus vergeselschaftete Nematodenarten. 2. Biologie et histologie de Dryocoetes hectographus et influence des nématodes du tube digestif. Entomophaga, 1957, 2, 253-269.

Schell S. C. : Tissue reactions of Blatella germanica L. to the developing larva of Physaloptera hispida Schell (Nematoda : Spiruroïdea). Trans. Am. Microcs. Soc., 1950, 71, 293-302.

SEUREAU C. : Données histologiques et ultrastructurales sur la réaction cellulaire de Locusta migratoria (Orthoptera : Acridien) parasité par Rictularia proni (Nematoda : Rictulariidae). C.R. Acad. Sci., D., Paris, 1972, 274, 44I-444.

Seureau C. : Réactions cellulaires provoquées par les nématodes Subulures et Spirurides chez Locusta migratoria (Orthoptère) : Jocalisation et structure des capsules. Z. Parasitenk., 1973, $4 I, 119-138$.

TAYLOR R. L. : Tissue damage induced by an oxyuroid nematode, Leidynema sp. in the hind gut of the Madeira cockrach, Leucophaea maderae. J. Invert. Pathol., I968, 11, 214-218.

WELCH H. E. : Entomophilic nematodes. A Rev. Entomol., 1965, 10, 275-302. 